\title{
H. pylori Antigen Detection in Stool
}

Dear Editor,

H. pylori antigen detection in stool is a rapid, non invasive, easy to perform test that can be used to detect active infection, monitor effectiveness during therapy and to confirm cure after antibiotic use. ${ }^{1}$ The ease to collect the specimen, especially in children, in whom endoscopy would be difficult and the non requirement of specially trained staff to collect and perform the test adds to the tests advantage. Also, prior preparation of the patient is not necessary unlike in upper gastrointestinal endoscopy. The sensitivity and specificity of this test to the other detection methods of $H$. pylori have been confirmed and documented. ${ }^{2}$

We tested stool samples from 36 cases of whom 12 had dyspepsia, 15 endoscopically proven antral gastritis, six cancer stomach and three healthy volunteers. The age of the patients ranged from 7 to 75 years and as we had difficulty collecting stool specimens from patients prepared for endoscopy, follow up was not possible in all cases, and we resorted to availability or convenience sampling.

We used the H.pylori antigen (Hpag) detection kit manufactured by Premier Platinum HPSA, Meridian Bioscience, to detect H.pylori antigen in stool. Hpag was detected in three out of 12 patients with dyspepsia, 12 out of 15 patients with antral gastritis, four out of six patients with cancer stomach and one out of three voulnteers. Thus, Hpag was detected in all groups. The test is very useful in follow up and repeated endoscopies can be avoided. Suspected childhood risk factors for $H$. pylori infection include having a single parent, lower parental social class, greater housing density or proportion of rented housing in school catchment area, overcrowding, bed-sharing, and absence of a fixed hot water supply. In adults, previously investigated risk factors include race-ethnicity, socioeconomic status, education, dental prosthesis and irregular dental treatment, public contact, working with animals, smoking status, alcohol consumption, homosexuality, sexually transmitted diseases, and having an infected child or partner. ${ }^{3}$ This test makes it convenient to determine the epidemiology of H.pylori in children.

Comparative accuracy, availability, and cost of tests for $H$ pylori infection ${ }^{4}$ puts the sensitivity and specificity of the stool antigen test at $90-95 \%$ for each, thus making it the most preferred investigation. However, lack of awareness of the existence of the stool antigen tests and non availability of this test in many places, makes physicians to opt for other test procedures.

\section{References}

1. Vaira D, Vakil N, Menegatti M, Van't Hoff B, Ricci C, Gatta L, et al. The stool antigen test for detection of Helicobacter pylori after eradication therapy. Ann Intern Med 2002;136:280-7.

2. Vaira D, Malfertheiner P, Mergraud F, Axon AJ, Deltenre M, Gasbarrini G, et al. Noninvasive antigen-based assay for assessing Helicobacter pylori eradication; a European 
multicenter study. The European Helicobacter pylori HpSA study group. Am J Gastroenterol 2000;95:925-9.

3. Hunt R. Helicobacter pylori: from theory to practice. Am J Med 1996;100:1-64.

4. Logan RP, Walker MM. ABC of the upper gastrointestinal tract: epidemiology and diagnosis of Helicobacter pylori infection. $\mathrm{Br}$ Med J 2001;323:920-2.
JJ Martin, N Anbumani, M Kalyani, *PK Rajesh Department of Microbiology, Sri Ramachandra Medical College \& Research Institute, Porur, Chennai- 600 116, Tamilnadu, India.

*Corresponding author (email: <dr_pkrajesh@hotmail.com>)

Received: 26-06-05

Accepted: 27-07-05 